\title{
Assessing the magnitude of reporting bias in systematic reviews \\ - A cross-sectional comparison between PROSPERO and Cochrane samples
}

Authors:

Maximilian Siebert (1,2), Laura Caquelin (1,2), Meisser Madera (3), Roberto Acosta-Dighero (4), Florian Naudet (1), Marta Roqué $(5,6)$

Affiliations:

(1) Univ Rennes, CHU Rennes, Inserm, CIC 1414 [(Centre d'Investigation Clinique de Rennes)], F-35000 Rennes, France

(2) Univ Rennes, CHU Rennes, REPERES [(Recherche en Pharmaco-épidémiologie et Recours aux Soins)], EA 7449, 35000 Rennes, France

(3) Department of Research, Faculty of Dentistry at the University of Cartagena, Cartagena, Colombia

(4) School of Physiotherapy, Faculty of Health Sciences Universidad San Sebastián, Santiago, Chile

(5) Iberoamerican Cochrane Centre - Sant Pau Biomedical Research Institute (IIBSant Pau), Barcelona, Spain

(6) CIBER of Epidemiology and Public Health (CIBERESP), Madrid, Spain

ORCID numbers:

Maximilian Siebert: 0000-0003-4385-5773

Laura Caquelin: 0000-0003-4557-3315

Meisser Madera: 0000-0002-2350-4194

Roberto Acosta-Dighero: 0000-0002-2372-6088

Florian Naudet: 0000-0003-3760-3801

Marta Roqué: 0000-0003-0043-1364

Corresponding Author:

Maximilian Siebert, Clinical Investigation Center (Inserm 1414), Rennes University Hospital, Rennes, France

Maximiliansiebert91@gmail.com 


\section{Abstract:}

Objective: To explore differences between published reviews and their respective protocols in a sample of 97 non-Cochrane systematic reviews (non-CSRs) and 97 Cochrane systematic reviews (CSRs) in terms of PICOS elements and the extent to which they were reported.

Study Design and Setting: We searched PubMed and Cochrane databases to identify nonCSRs and CSRs that were published in 2018. We then searched for their corresponding Cochrane or PROSPERO protocols. The published reviews were compared to their protocols. The primary outcome was changes from protocol to review in terms of PICOS elements.

\section{Results:}

More than half of each sub-sample (54.6\% of CSRs \& $67 \%$ of non-CSRs) (Absolute Risk Reduction (ARR) 12.6\% [-1.3\%; 26\%]) presented changes in PICOS elements. A total of 227 changes were identified, 108 (47.5\%) in CSRs and 119 (52.5\%) in non-CSRs. For both subsamples, approximately a third of all changes corresponded to changes related to primary outcomes. Marked differences were found between the sub-samples for the reporting of changes. 95.8\% of the changes in PICOS items were not reported in the non-CSRs compared to $42.6 \%$ in the CSRs (ARR 53.2\% [43.2\%; 63.2\%]).

Conclusion: CSRs showed better results than non-CSRs in terms of the reporting of changes. Reporting of changes from protocol needs to be promoted and requires general improvement. The limitations of this study lie in its cross-sectional nature.

Registration: https://osf.io/6j8gd/

6 keywords: Methodology; Cochrane; Systematic Review; Protocol; PROSPERO; Transparency 


\section{Introduction}

Systematic Reviews (SRs) play an essential role inside and outside biomedical research. Because of their high standing in the pyramid of evidence, their results can change how medical research is conducted, how clinical decisions are reached, how policies are designed, and how patients are informed about the benefits and risks of therapeutic interventions. (1-3).

SRs in the Cochrane framework are pivotal in this process. This is linked to the development of helpful guidelines and tools for the conduct of SRs, such as bias assessments, and the publication of the Cochrane Handbook (4). Cochrane Systematic Reviews (CSRs) are often described as the gold standard of SRs and enjoy a high level of confidence $(5,6)$

The pre-registration of protocols is considered as methodologically important and especially so because it is considered as an instrument to avoid reporting bias (7). Furthermore, it increases transparency in the details provided on the research and on subsequent changes from the methods planned. Thus peer-reviewers are able to consult the protocol and get a better understanding of the research project. Today, pre-registrations have reached such standing that several journals, such as the PLoS journals or the BMJ, include the registration details in their instructions to future authors, who then incorporate them in the final manuscripts (8) (9).

In addition to the Cochrane Database of Systematic Reviews (CDSR), SRs focusing on health outcomes can be prospectively registered on PROSPERO, which was launched in 2011 as a free, open, online tool, where researchers can register their protocols in a database before starting the actual SR (9). Besides the initiative to promote open research, this platform has the advantage of assigning each protocol to a unique identifier to facilitate its identification and transparency.

Following the above-mentioned initiatives, numerous studies have been able to assess discrepancies between the original protocols and the published SRs in the biomedical field for both CSRs and non-Cochrane SRs (non-CSRs)(10-12).

However, most studies that have looked at the differences between the registered protocols and the published SRs have focused on changes in primary outcomes (11-13). While these are paramount characteristics in a SR, all the elements that form the PICOS method (Patients, Intervention, Comparison/control, Outcome and Study type) are equally important.

Therefore, changes in any of these elements could have a major impact on the final SR and thus reporting these changes is crucial to ensure transparency and avoid reporting biases. Aware of these issues, Cochrane updated its Handbook in 2008 and 2013 accordingly. Since 2008, a requirement to report changes from protocol to review have been included, and since 2013, any changes in the published SRs need to be justified $(4,12)$.

The aim of this study was to investigate whether the new measures have brought any improvement in the quality of CSRs by reducing the discrepancies between CSR protocols and SRs, as compared to SRs published elsewhere and not constrained by such requirements. To this end, we assessed the frequency and reporting of changes in PICOS elements from protocol to SR and the reporting of these changes, in a sample of SRs published in 2018, and compared the results to non-CSRs registered on PROSPERO. 


\section{Methods}

\subsection{Study Design and Registration}

The design of this study was cross-sectional, comparing CSRs and non-CSRs published in 2018 to their pre-registered protocols, with a focus on the PICOS method. The aims and the methods of this study were described prior to the study in a protocol registered on 25/09/2019 on the Open Science Framework: https://osf.io/6j8gd/

\subsection{Inclusion Criteria}

We included SRs with or without meta-analyses assessing any therapeutic intervention in any disease, widely known as intervention SRs (4), if they had a registered protocol on either CDSR or PROSPERO, and were published for the first time between 01/01/2018 and 01/01/2019. For each SR selected, the protocol was retrieved. SRs and their protocols were to be published in English. When several versions of the same SR were identified, we only considered the most recent. SRs on diagnosis, prognosis, safety, or cost-effectiveness were excluded.

\subsection{Electronic searches}

We searched for CSRs in the Cochrane Database of Systematic Reviews (via the Cochrane Library). This database contains all CSRs and features a tool that enables searches according to review type and publication date. We used these filters to identify potential intervention CRs to be included in our study. This search was conducted on 02/09/2019.

Likewise, we searched non-CSRs (PROSPERO SRs) on Medline (via PubMed). In order to identify non-CSRs on the intervention type from this database, we used a search strategy designed by an expert in this field (supplementary material Table 1). In brief, it enables the identification of SRs on PubMed that have a PROSPERO CDR registration number. This number then enables the retrieval of the original protocol on PROSPERO. This search was conducted on 10/09/2019.

\subsection{Selection of reviews}

For the CSRs, we imported all retrieved records on an Excel sheet and randomized them. Similarly, for the non-CSRs, one author (M.S.) screened the results of the search by title and abstract, to exclude irrelevant studies. Full-text SRs were obtained for further assessment to determine final inclusion according to the eligibility criteria. A random selection of the sample was performed with the r-norm function in R (14).

\subsection{Hypotheses \& sample size calculation}

We hypothesized that there would be $20 \%$ fewer CSRs reporting major changes from protocol to review, on the basis of the published literature. We identified $40 \%$ of CSRs presenting differences from protocol to SR for outcomes (12) and around 60\% of non-CSRs presenting 
differences for the eligibility criteria (15). The alpha error retained was 0.05 in combination with a bilateral hypothesis test. We calculated that for the two sub-samples we needed a total of 194 SRs (97 CSRs and 97 non-CSRs) to reach a power of $80 \%$ to test our hypotheses.

\subsection{Quality assessment of the SRs included}

To assess the methodological quality of the SRs, we used the A Measurement Tool to Assess Systematic Reviews -2 (AMSTAR-2). It is composed of 16 items, among which seven are critical domains. There are four levels to be distinguished: high, moderate, low and critically low (16).

The quality of SRs was assessed by at least one reviewer (M.S. or M.R.).

\subsection{Data collection}

Before data collection, all reviewers (M.S., L.C., R.D. or M.M.) completed a pilot sample of 3 studies for each review type. At least two of the reviewers extracted data independently, and any discrepancies were resolved by consensus. In case of disagreement, a third reviewer arbitrated (M.R.).

We extracted the following general characteristics of the SRs: changes in protocol title and SR title, changes in first author between protocol and SR, country of affiliation of first author, country of affiliation of corresponding author, publication date of protocol, dates of changes/ searches (if indicated), publication date of SR.

Furthermore, any changes from protocol to SR on PICOS items were collected. Finally changes in secondary outcomes and reporting of the changes from protocol to SR were explored.

Our primary outcome was change from protocol to review in terms of PICOS elements. Our secondary outcomes were changes from protocol to SR in the secondary outcomes, the reporting in the published SRs of changes, the time between protocol and SR publication, the time between the last searches and SR publication, and SR quality.

For any given review, all changes in PICOS characteristics were assessed in terms of the magnitude of change via an ad-hoc exploratory scale that classified changes into small, moderate, or major in terms of their relevance within the SR (see Table 1). By applying this scale, we gave priority to crucial changes, such as changes in the population reported in the final article, over the reporting of less important changes from protocol. The classification was conducted in agreement between researchers on the perceived relevance of the change in relation to the review.

\begin{tabular}{|l|l|}
\hline \multicolumn{1}{|c|}{ Magnitude of Change } & \multicolumn{1}{c|}{ Examples } \\
\hline Small & $\begin{array}{l}\text { - changing the definition of an outcome/intervention, but only to } \\
\text { make it clearer or more complete }\end{array}$ \\
\hline Moderate & $\begin{array}{l}\text { adding or deleting a secondary outcome } \\
- \text { changing study type inclusion criteria }\end{array}$ \\
\hline
\end{tabular}




\begin{tabular}{|c|c|}
\hline & $\begin{array}{l}\text { reporting only on severe adverse effects, although separate } \\
\text { reporting for general adverse effects and severe adverse effects } \\
\text { was planned }\end{array}$ \\
\hline Major & $\begin{array}{l}\text { - adding or eliminating a primary outcome } \\
\text { - setting up composite endpoints instead of individual ones } \\
\text { - changing a comparator intervention } \\
\text { - changing the population of interest in the SR }\end{array}$ \\
\hline
\end{tabular}

Table 1. Examples of the classification of changes

\subsection{Data management and synthesis}

Frequencies and percentages were used for categorical variables and means and standard deviations with the corresponding $95 \%$ confidence intervals, were used for continuous variables. For proportions, we used the Chi-square test, and for means, we ran a MannWhitney U test.

Estimates of association were computed between time in months from protocol to SR and primary outcome changes from protocol to SR concerning population, comparisons and outcomes.

The shortfall between non-CSRs and CSRs in terms of changes and their reporting was assessed with the Absolute Risk Reduction (ARR) method and the respective 95\% Confidence Interval.

Corresponding $\mathrm{p}$-values were calculated to find out whether there was a statistically significant difference between the CSR and the non-CSR sub-samples. The analyses were conducted on R Studio (17).

\subsection{Deviations from protocol to SR publication}

The deviations from protocol to SR publication can be found in the supplementary material.

\section{Results}

\subsection{Search results and description of the sample}

The selection process is reported in Figure 1. Briefly, for CSRs, we identified 339 records. Of these, $310(91.4 \%)$ were considered as intervention results. A total of 97 SRs were randomly selected and included in our analyses. Likewise, for non-CSRs, our systematic literature search retrieved 648 records. Of these, 397 were excluded, mainly for not being interventional SRs, resulting in a total of 251 (38.7\%) eligible SRs, from which the final sample of 97 non-CSRs was randomly selected.

Out of the 194 SRs included, more than $70 \%$ in each sample had a first author from a highincome region: $78.4 \%$ among CSRs and $71.1 \%$ among non-CSRs. The same was true for the corresponding authors $(78.4 \%$ and $72.2 \%$, respectively). 
Time between the publication of the protocol and the final published review was 31.4 months on average ([95\%CI $28-34.9])$. CSRs and non-CSRs differed markedly in the time between the publication of the protocol and the final SR, which was longer for CSRs (41.1 months [35.2-47.1]) than for non-CSRs (21.8 months [19.2-24.4]) (p-value <0.01). Conversely, the time between the date of publication and the last amendments to the protocol was shorter for CSRs (1.4 [0.3-3.1]) than for non-CSRs (13.1 [9.5-16.8]) (p-value <0.01).

\subsection{Comparison of protocols with their published systematic reviews}

Overall, our research yielded 32 CSRs (33\%) that presented no change between protocol and SR, $53(54.6 \%)$ that included a change in PICOS elements, and $12(12.3 \%)$ that included other changes. For the 53 CSRs that involved changes in PICOS elements, more than half (28 SRs, 52,8\%) presented at least one major change. Out of these 28 SRs, 10 (35.7\%) reported all changes (major, moderate and small), and 19 (67.9\%) reported all major changes.

Among the non-CSRs, we found 24 SRs, $(24.7 \%)$ that presented no changes from protocol, $65(67 \%)$ that had changes in PICOS elements, and 8 SRs with no changes in PICOS outcomes. Concerning the 65 remaining SRs, 39 SRs (60\%) presented at least one major change; of these 39 SRs, none reported all changes and only $2(5.1 \%)$ reported all major changes.

Quantification of the changes in PICOS elements made it possible to identify a total of 227 changes, 108 (47.5\%) in the CSRs and 119 (52.5\%) in the non-CSRs.

Following breakdown of the distribution of the changes according to the PICOS elements, we observed that for both samples approximately a third of the total changes corresponded to changes related to primary outcomes. These results can be found in Figure 2.

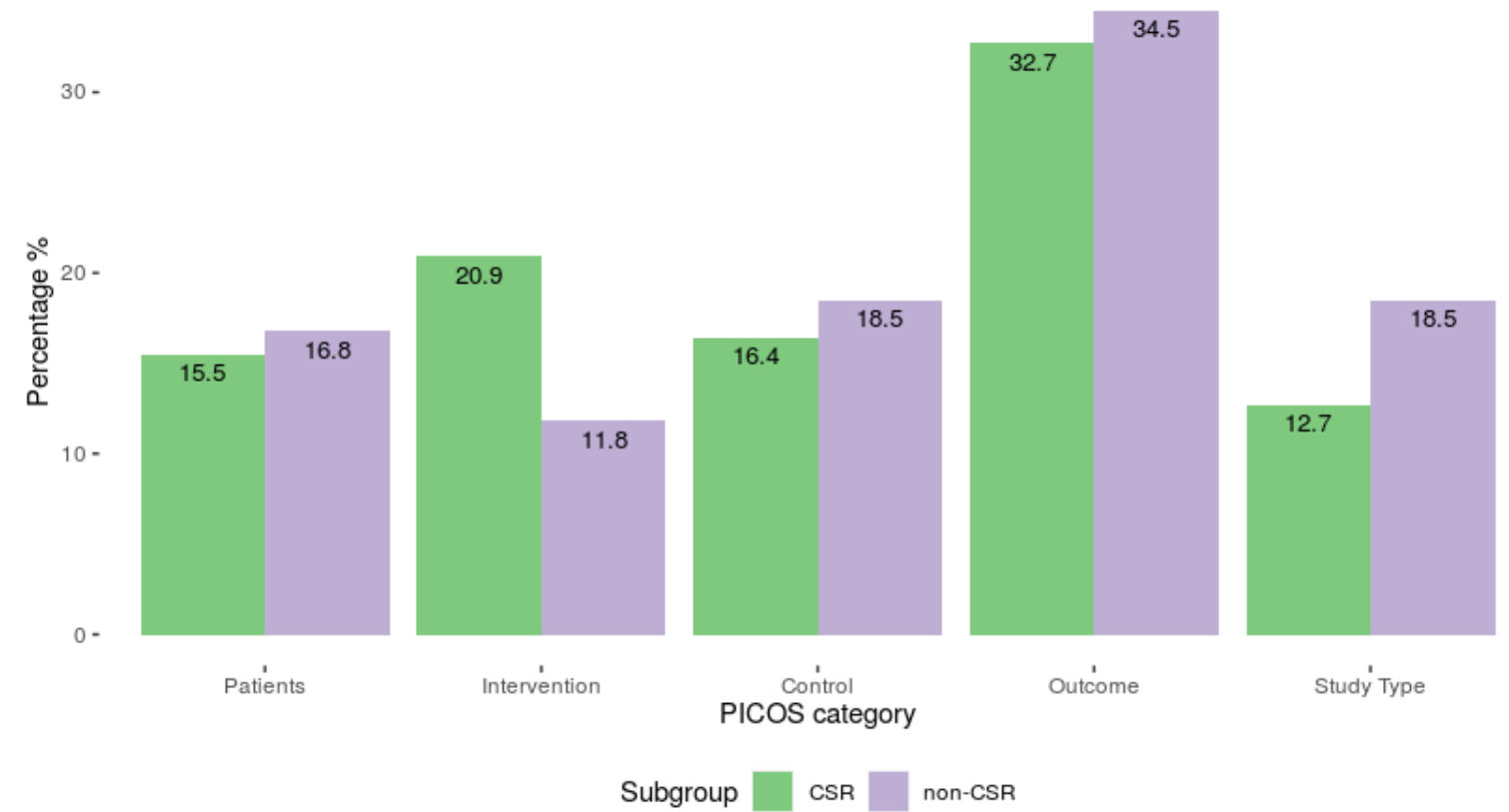


Figure 2. Distribution of changes according to PICOS elements and Review Type

With respect to the magnitude of change, in CSRs the largest proportion of the 108 changes in the CSRs were small (42.6\%), and major changes made up approximately a third of the total $(32.4 \%)$. Non-CSRs had the largest share of changes in the major change section, with a percentage of $38.7 \%$.

Concerning the reporting of changes, in all $42.6 \%$ of CSRs and $95.8 \%$ of non-CSR changes were not reported (ARR 53.2\% [43.2\%; 63.2\%]). For both samples, the lowest reporting rate was found for small changes.

Details of the percentages, ARR-values and p-values for changes in relation to the magnitude of changes and the reporting of changes are shown in Table 2.

\begin{tabular}{|c|c|c|c|c|c|}
\hline $\begin{array}{c}\text { Total changes in } \\
\text { PICOS }\end{array}$ & $\begin{array}{c}\text { CSRs } \\
(\mathrm{n}=108)\end{array}$ & $\begin{array}{c}\text { Non-CSRs } \\
(\mathrm{n}=119)\end{array}$ & ARR & 95\%CI & $\begin{array}{c}\text { p-Value } \\
\text { (Chi-square) }\end{array}$ \\
\hline Small changes & $46(42.6 \%)$ & $34(28.6 \%)$ & $-14 \%$ & {$[-26.4 \% ;-1.7 \%]$} & 0.04 \\
\hline Not Reported & $24(52.2 \%)$ & $34(100 \%)$ & $47.8 \%$ & {$[33.4 \% ; 62.3 \%]$} & $<0.01$ \\
\hline Moderate changes & $27(25 \%)$ & $39(32.7 \%)$ & $7.7 \%$ & {$[-4 \% ; 19.5 \%]$} & 0.25 \\
\hline Not Reported & $12(44.4 \%)$ & $36(92.3 \%)$ & $47.9 \%$ & {$[27.3 \% ; 68.4 \%]$} & $<0.01$ \\
\hline Major changes & $35(32.4 \%)$ & $46(38.7 \%)$ & $6.3 \%$ & {$[-6.2 \% ; 18.7 \%]$} & 0.40 \\
\hline Not Reported & $10(28.6 \%)$ & $44(95.7 \%)$ & $67.1 \%$ & {$[51 \% ; 83.2 \%]$} & $<0.01$ \\
\hline
\end{tabular}

Table 2. Distribution of changes in PICOS elements according to magnitude of change, reporting status and review type

With respect to changes not related to PICOS elements, of the 97 CSRs included, $21.6 \%$ presented a change in first author, compared to non-CSRs with 22.7\%; (ARR 1.1\% [-10.7\%; $12.7 \%]$ ), the difference being non-significant. For changes in the SR title, 30.9\% of CSRs and $55.7 \%$ of non-CSRs (ARR 24.8\% [11.2\%;38.2\%] presented a change.

Regarding the secondary endpoints, we identified one or more changes in 39 of the 97 CSRs (40.2\%) and in 43 of the 97 non-CSRs (44.3\%) (ARR 4.1\% [-9.8\%; 18\%]). Moderate changes were observed more often in non-CSRs than in CSRs (93\% vs. 76.9\%) (ARR 16.1\% $[0.8 \% ; 31.4 \%])$ and small changes appeared less often because we only distinguished these two types of changes. Non-reporting of changes in the secondary outcomes, without distinction for magnitude, was considerably more frequent among the non-CSRs, with only one non-CSR reporting any compared to more than half the CSRs reporting changes $(97.7 \%$ vs. $48.7 \%$ (ARR 49\% [32.6\%; 65.3\%]). 


\subsection{Methodological quality of the reviews in relation to changes}

For the methodological quality of the SRs, $100 \%$ of CSRs achieved the highest ranking, while the quality of the non-CSRs was heterogeneous, with over half (50.5\%) classified as low quality and $21.6 \%$ as critically low.

Our results are shown in Figure 3.

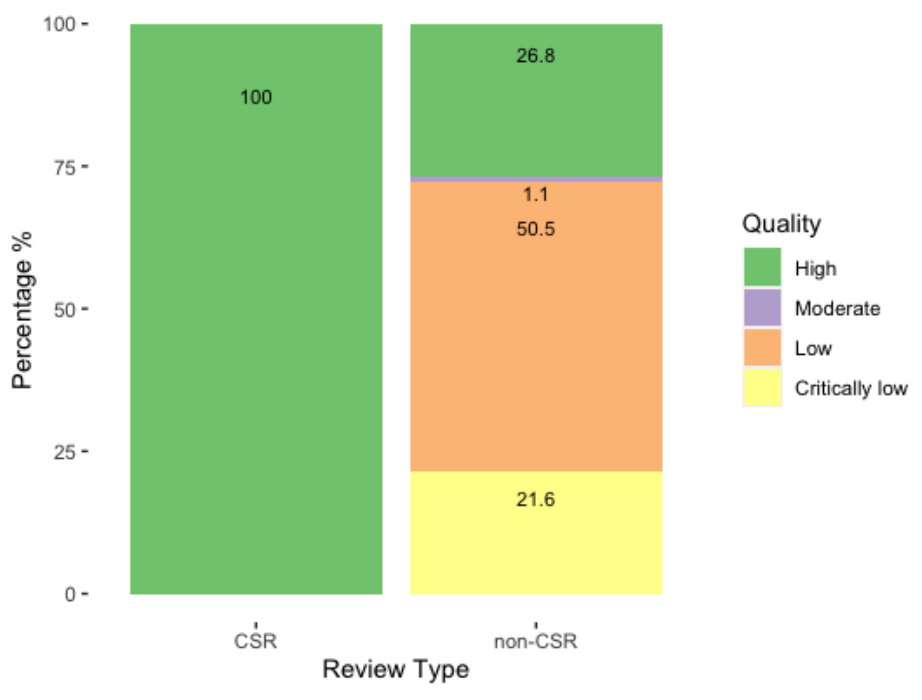

Figure 3. Quality of SRs assessed by AMSTAR-2

There was a clear association between lower methodological quality of non-CSRs and the occurrence of changes from protocol, with lower-quality reviews being more likely to present changes than higher-quality reviews. The same pattern was found across the four possible magnitudes of change (Supplementary material Figure 1).

Methodological quality was also related to the reporting of changes from protocol. Non-CSRs with non-reported changes were mostly of low quality (Supplementary material Figure 2).

\section{Discussion}

\subsection{Summary of main findings}

In this study we compared 97 non-CSRs and 97 CSRs that focused on any therapeutic interventions and were published in 2018. Changes between the protocols and the published 
SRs were described in terms of PICOS elements, non-PICOS elements and secondary outcomes.

More than half of each sub-sample, $54.6 \%$ of CSRs and $67 \%$ of non-CSRs presented changes in PICOS elements (ARR 12.6\% [-1.3\%; 26\%]). Furthermore, we found that most changes related to the primary outcome whatever the type of SR.

Overall, we found similar proportions for major changes in CSRs and non-CSRs (32.4\% vs $38.7 \%$ ) (ARR 6.3\% [-6.2\%;18.7\%]). However, manifest differences were found between the sub-samples with regard to the reporting of changes. Only $4.2 \%$ of changes in PICOS items were reported in non-CSRs, compared to $57.4 \%$ in CSRs (ARR 53.2\% [43.2\%; 63.2\%]). A similar pattern was observed with regard to changes reported for the secondary outcomes, $51.3 \%$ in CSRs and 2.3\% in non-CSRs (ARR 49\% [32.6\%; 65.3\%]).

Finally, we observed a significant difference in the occurrence of small changes, with CSRs presenting more numerous changes than non-CSRs (42.6\% vs. 28.6\%) (ARR -14\% [-26.4\%; $-1.7 \%])$.

These finding suggest that there are still many deviations from registered protocols that are not described or justified in published SRs. Applying the AMSTAR-2 tool, we observed $100 \%$ high quality classification for CSRs, whereas more than two-thirds $(72.1 \%)$ of nonCSRs were classified low or even critically low quality.

\subsection{Comparison of our findings with other studies}

Several studies have focused only on certain outcomes in either non-CSRs or CSRs (13, 18, 19) and studies comparing the two sub-groups are rare. In addition, we adopted a new type of approach and created our own Likert scale to assess the magnitude of changes. Therefore, it is somewhat difficult to compare our findings to other studies. Nevertheless, we have endeavoured to put our research in context by comparing partial results to other papers.

In a study in 2014, Page and colleagues conducted a meta-analysis of four studies including 485 CSRs, found that $38 \%$ of the studies made a change to at least one primary outcome (20). Our numbers are similar to these findings in 2014, although Page's results were only valid for CSRs, and the study was conducted before the requirements of reporting of changes by Cochrane was mandatory.

Koensgen and colleagues checked for several PRISMA items when comparing protocol and final SR, and they did not focus solely on primary outcomes. However, they explored only non-CSR reviews (15). $92.5 \%$ of the reviews involved a change, almost $50 \%$ included a major change for an item in the PRISMA list and only $10 \%$ of the changes were reported. The percentage of changes reported in non- CSRs in this study was higher than in ours $(10 \%$ vs. $4.2 \%$ ). One difference from our approach was the distinction between major and minor changes only, whereas we introduced a third category of moderate changes. Consequently, we present a lower rate of major changes in non-CSRs. While the definitions vary, the findings show that there is a real issue with the reporting of major changes in non-CSRs.

In a recent study by $\mathrm{Hu}$ and colleagues, which focused on changes in PICOS outcomes from protocol to review in PROSPERO-registered reviews, the researchers found that $90 \%$ of the reviews underwent one change and 59\% at least two changes. In line with our results, they 
found very low rates for reporting of changes and observed that the main area in SRs prone to changes concerned the outcomes (21).

The conclusions of the above-mentioned studies confirm our impression that changes are insufficiently reported and that there is a huge difference for SRs produced outside the Cochrane framework. Furthermore, they show that the numbers of changes in primary outcomes have remained at the same level over recent years. Creating awareness of the lack of reporting on deviations from protocol should thus be a priority.

\subsection{Strengths and limitations}

Among the strengths of this study, we would like to stress that this was a first approach to the research question, in the form of a cross-sectional study including a representative sample of non-CSRs and CSRs. Furthermore, a sensitive search strategy was performed to identify nonCSRs. In addition, more than one reviewer independently conducted the whole selection process and data extraction from the SRs included. We also included an objective, widely used tool for assessing the quality of the SRs. We did not only focus on primary and secondary outcomes only, but we tried to have a more inclusive overview by observing PICOS characteristics. Finally, all our methods were specified a priori in a protocol.

Nonetheless, certain limitations to this study should be considered. Firstly, due to lack of resources, we were not able to contact all the authors and confront them with our findings which would have cleared some issues. Secondly, detecting changes was easier when they were reported, as was the case for CSRs.

Indeed, the data extractors (mainly early career researchers) may have judged changes too strictly in some cases, and they may not have been experienced enough to know how difficult it is to be transparent in research. This also applies to the AMSTAR-2 assessment of SR quality. We only included SRs in English. Had other languages been included, our analysis might have looked different.

Finally, our identification of changes in PICOS factors between protocols and SRs in a crosssectional study setting is only exploratory. Numerous unmeasured confounders, for example the journals' word count restrictions or unclear author instructions, could account for some of the associations found. There may be other confounders, and great caution is warranted in interpreting these results.

\subsection{Implications for practice}

We would encourage journals and publishers to generate recommendations that provide for differences from protocol to review to be indicated. Should the patterns observed in our investigation persist, reporting biases, unpublished studies and unrealistic research standards will result. A possible solution to the issue could be that journals assign one peer-reviewer to the task of checking the differences in relation to the protocols (22).

\section{Conclusions}

The reporting of differences between protocol and publication in CSRs and non-CSR has not improved compared to earlier studies. Despite new rules in the Cochrane framework and 
initiatives such as PROSPERO, there is room for improvement. We are aware that a new investigation on this topic is currently being undertaken, not restricted to PROSPERO alone and with a properly registered CSR review (23). We hope that our findings and future research will improve reporting quality regarding changes between protocol and review.

Acknowledgments:

We would like to thank Ivan Solà from the Iberoamerican Cochrane Centre for helping us with the search strategy designed to retrieve SRs that had a PROSPERO-registered protocol.

Conflicts of interest:

Marta Roqué, Roberto Acosta-Dighero \& Meisser Madera are members of Cochrane. Maximilian Siebert did a 2-month internship at the Iberoamerican Cochrane Center in 2019.

Data-sharing agreement:

All data is available and can be found under this link: https://osf.io/5ywus/

\section{References}

1. Mulrow CD. Rationale for systematic reviews. BMJ. 1994;309(6954):597-9.

2. Murad MH, Asi N, Alsawas M, Alahdab F. New evidence pyramid. Evidence Based Medicine. 2016;21(4):125-7.

3. Systematic Reviews: Synthesis of Best Evidence for Clinical Decisions. Annals of Internal Medicine. 1997;126(5):376-80.

4. Higgins JPT, Thomas J, Chandler J, Cumpston M, Li T, Page MJ, et al., editors. Cochrane Handbook for Systematic Reviews of Interventions version 6.22021.

5. Useem J, Brennan A, LaValley M, Vickery M, Ameli O, Reinen N, et al. Systematic Differences between Cochrane and Non-Cochrane Meta-Analyses on the Same Topic: A Matched Pair Analysis. PloS one. 2015;10(12):e0144980-e.

6. Rosenbaum SE, Glenton C, Cracknell J. User experiences of evidence-based online resources for health professionals: user testing of The Cochrane Library. BMC Med Inform Decis Mak. 2008;8:34-.

7. GC R, IJ O. Reporting bias 2019 [Available from:

https://catalogofbias.org/biases/reporting-biases/.

8. Stewart L, Moher D, Shekelle P. Why prospective registration of systematic reviews makes sense. Syst Rev. 2012;1:7.

9. York Uo. About PROSPERO 2020 [Available from:

https://www.crd.york.ac.uk/prospero/\#aboutpage.

10. Silagy CA, Middleton P, Hopewell S. Publishing Protocols of Systematic

ReviewsComparing What Was Done to What Was Planned. JAMA. 2002;287(21):2831-4.

11. Tricco AC, Cogo E, Page MJ, Polisena J, Booth A, Dwan K, et al. A third of systematic reviews changed or did not specify the primary outcome: a PROSPERO register study. J Clin Epidemiol. 2016;79:46-54. 
12. Pandis N, Fleming PS, Worthington H, Dwan K, Salanti G. Discrepancies in Outcome Reporting Exist Between Protocols and Published Oral Health Cochrane Systematic Reviews. PLoS One. 2015;10(9):e0137667.

13. Dwan K, Kirkham JJ, Williamson PR, Gamble C. Selective reporting of outcomes in randomised controlled trials in systematic reviews of cystic fibrosis. BMJ Open.

2013;3(6):e002709.

14. R Core Team. R: A language and environment for statistical computing. Vienna, Austria: R Foundation for Statistical Computing; 2018.

15. Koensgen N, Rombey T, Allers K, Mathes T, Hoffmann F, Pieper D. Comparison of non-Cochrane systematic reviews and their published protocols: differences occurred frequently but were seldom explained. J Clin Epidemiol. 2019;110:34-41.

16. Shea BJ, Reeves BC, Wells G, Thuku M, Hamel C, Moran J, et al. AMSTAR 2: a critical appraisal tool for systematic reviews that include randomised or non-randomised studies of healthcare interventions, or both. BMJ. 2017;358:j4008.

17. Team. R. RStudio: Integrated Development for R. Boston, MA: RStudio, PBC; 2020.

18. Kirkham JJ, Altman DG, Williamson PR. Bias due to changes in specified outcomes during the systematic review process. PLoS One. 2010;5(3):e9810.

19. Parmelli E, Liberati A, D'Amico R. Reporting of outcomes in systematic reviews: comparison of protocols and published systematic reviews. 15th Cochrane Colloquium; 2007; Sao Paolo2007.

20. Page MJ, McKenzie JE, Kirkham J, Dwan K, Kramer S, Green S, et al. Bias due to selective inclusion and reporting of outcomes and analyses in systematic reviews of randomised trials of healthcare interventions. Cochrane Db Syst Rev. 2014(10).

21. Hu K, Zhao L, Zhou Q, Mei F, Gao Q, Chen F, et al. Inconsistencies in study eligibility criteria are common between non-Cochrane systematic reviews and their protocols registered in PROSPERO. Research Synthesis Methods. 2021;12(3):394-405.

22. Thibault R, Thompson J, Munafò M, Katie Drax K, Clark R. A peer review intervention to minimize discrepancies between preregistrations and published manuscripts. Reward Equator Conference; 2020. Berlin2020.

23. Pieper D, Allers K, Mathes T, Hoffmann F, Klerings I, Rombey T, et al. Comparison of protocols and registry entries to published reports for systematic reviews. Cochrane Db Syst Rev. 2020(2). 

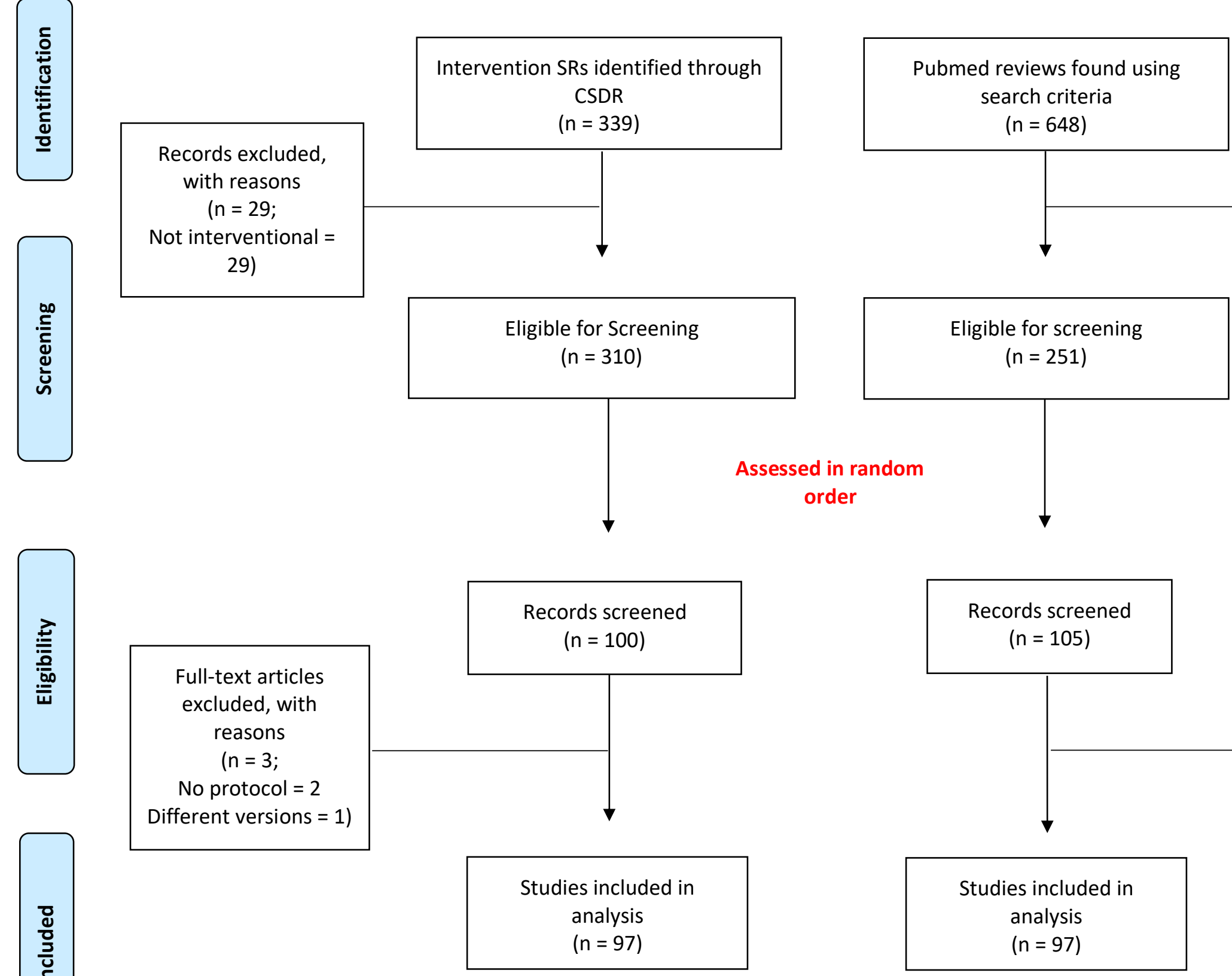

Records excluded, with reasons $(\mathrm{n}=397$;

Only protocol $=43$

Not systematic $=34$

No review $=42$

Not PROSPERO $=16$

Not interventional $=260$

Not in English = 2)

Figure 1: Flowchart of our selection process for Systematic Reviews 
